\title{
Analytic semigroups generated by Dirichlet-to-Neumann operators on manifolds
}

\section{Tim Binz ${ }^{1}$}

Received: 23 January 2020 / Accepted: 27 March 2021 / Published online: 18 May 2021

(c) The Author(s) 2021

\begin{abstract}
We consider the Dirichlet-to-Neumann operator associated to a strictly elliptic operator on the space $\mathrm{C}(\partial M)$ of continuous functions on the boundary $\partial M$ of a compact manifold $\bar{M}$ with boundary. We prove that it generates an analytic semigroup of angle $\frac{\pi}{2}$, generalizing and improving a result of Escher with a new proof. Combined with the abstract theory of operators with Wentzell boundary conditions developed by Engel and the author, this yields that the corresponding strictly elliptic operator with Wentzell boundary conditions generates a compact and analytic semigroups of angle $\frac{\pi}{2}$ on the space $\mathrm{C}(\bar{M})$.
\end{abstract}

Keywords Dirichlet-to-Neumann operator - Wentzell boundary conditions · Analytic semigroup $\cdot$ Riemmanian manifolds

Mathematics Subject Classification 47D06 · 34G10 - 47E05 · 47F05

\section{Introduction}

Differential operators with dynamic boundary conditions on manifolds with boundary describe a system whose dynamics consisting of two parts: a dynamics on the manifold interacting with an additional dynamics on the boundary. This leads to differential operators with so called Wentzell boundary conditions, see [17, Sect. 2].

On spaces of continuous functions on domains in $\mathbb{R}^{n}$ such operators have first been studied systematically by Wentzell [39] and Feller [26]. Later Arendt et al. [8] proved that the Laplace operator with Wentzell boundary conditions generates a positive, contractive $C_{0}$-semigroup. Engel [20] improves this by showing that this semigroup

Communicated by Abdelaziz Rhandi.

$凶 \operatorname{Tim}$ Binz

binz@mathematik.tu-darmstadt.de

1 Department Mathematics, Technical University of Darmstadt, Schlossgartenstrasse 7, 64289

Darmstadt, Germany 
is analytic with angle of analyticity $\frac{\pi}{2}$. Later Engel and Fragnelli [17] generalize this result to uniformly elliptic operators, however without specifying the corresponding angle of analyticity. For related work see also [11-14,22,23,38,39] and the references therein. Our interest in this context is the generation of an analytic semigroup with the optimal angle of analyticity.

As shown in $[9,17]$ this problem is closely connected to the generation of an analytic semigroup by the Dirichlet-to-Neumann operator on the boundary space. More precisely, based on the abstract theory for boundary perturbation problems developed by Greiner [25], it has been shown in $[9,17]$ that the coupled dynamics can be decomposed into two independent parts: a dynamics on the interior and a dynamics on the boundary. The first one is described by the differential operator on the manifold with Dirichlet boundary conditions while the second is governed by the associated Dirichlet-to-Neumann operator.

On domains in $\mathbb{R}^{n}$ the generator property of differential operators with Dirichlet boundary conditions is quite well understood, see [7,29,32]. On compact Riemannian manifolds with boundary it has been shown in [10] that strictly elliptic operators with Dirichlet boundary conditions are sectorial of angle $\frac{\pi}{2}$ and have compact resolvents on the space of continuous functions.

Dirichlet-to-Neumann operators have been studied e.g. by [30,32,37] and [36, App. C]. For the operator-theoretic context see, e.g., the work of Amann and Escher [4] and Arendt and ter Elst [5-7]. In particular, on domains in $\mathbb{R}^{n}$ Escher [19] has shown that such Dirichlet-to-Neumann operators generate analytic semigroups on the space of continuous functions, however without specifying the corresponding angle of analyticity. Finally, ter Elst and Ouhabaz [16] proved that this angle is $\frac{\pi}{2}$ and extended the result of Escher [19] to differential operators with less regular coefficients.

In this paper we study such Dirichlet-to-Neumann operators on the space of continuous functions on Riemannian manifolds and show that they generate compact and analytic semigroups of angle $\frac{\pi}{2}$ on the continuous functions.

We first explain our setting and terminology. Consider a strictly elliptic differential operator $A_{m}: D\left(A_{m}\right) \subset \mathrm{C}(\bar{M}) \rightarrow \mathrm{C}(\bar{M})$, as given in (4.3), on the space $\mathrm{C}(\bar{M})$ of continuous functions on a smooth, compact, orientable Riemannian manifold $\bar{M}$ with smooth boundary $\partial M$. Moreover, let $\frac{\partial^{a}}{\partial \nu^{g}}: D\left(\frac{\partial^{a}}{\partial \nu^{g}}\right) \subset \mathrm{C}(\bar{M}) \rightarrow \mathrm{C}(\partial M)$ be the outer conormal derivative, $\beta>0$ and $\gamma \in \mathrm{C}(\partial M)$. We consider $B:=-\beta \cdot \frac{\partial^{a}}{\partial \nu^{g}} f+\left.\gamma \cdot f\right|_{\partial M}$ : $D(B) \subset \mathrm{C}(\bar{M}) \rightarrow \mathrm{C}(\partial M)$, as in (4.4), and define the operator $A^{B} f:=A_{m} f$ with Wentzell boundary conditions by requiring

$$
f \in D\left(A^{B}\right) \quad: \Longleftrightarrow f \in D\left(A_{m}\right) \cap D(B) \text { and }\left.A_{m} f\right|_{\partial M}=B f
$$

For a continuous function $\varphi \in \mathrm{C}(\partial M)$ on the boundary the corresponding Dirichlet problem

$$
\left\{\begin{array}{l}
A_{m} f=0, \\
\left.f\right|_{\partial M}=\varphi,
\end{array}\right.
$$


is uniquely solvable by Corollary A.4. Moreover, by the maximum principle, see Theorem A.1, the associated solution operator $L_{0}: \mathrm{C}(\partial M) \rightarrow \mathrm{C}(\bar{M})$ is bounded. Then the Dirichlet-to-Neumann operator is

$$
N \varphi:=-\beta \frac{\partial^{a}}{\partial \nu^{g}} \cdot L_{0} \varphi \quad \text { for } \varphi \in D(N):=\left\{\varphi \in \mathrm{C}(\partial M): L_{0} \varphi \in D(B)\right\}
$$

That is, $N \varphi$ is obtained by applying the Neumann boundary operator $-\beta \frac{\partial^{a}}{\partial \nu^{g}}$ to the solution $f$ of the Dirichlet problem (1.2).

Our main results are the following.

(i) The Dirichlet-to-Neumann operator $N$ in (1.3) generates a compact and analytic semigroup of angle $\frac{\pi}{2}$ on $\mathrm{C}(\partial M)$;

(ii) the operator $A^{B}$ with Wentzell boundary conditions (1.1) generates a compact and analytic semigroup of angle $\frac{\pi}{2}$ on $\mathrm{C}(\bar{M})$.

This extends the results from Escher [19] and Engel-Fragnelli [17, Cor. 4.5] to elliptic operators on compact manifolds with boundaries and gives the maximal angle of analyticity $\frac{\pi}{2}$ in both cases. In the flat case the result for the Dirichlet-to-Neumann operator coincides with the result of ter Elst-Ouhabaz [16] in the smooth case. The techniques here are different and our proof is independent from theirs.

This paper is organized as follows. In Sect. 2 below we recall the abstract setting from $[9,17]$ needed for our approach. Based on [20, Sect. 2], we study in Sect. 3 the special case where $A_{m}$ is the Laplace-Beltrami operator and $B$ the normal derivative. In Sect. 4 we then generalize these results to arbitrary strictly elliptic operators and their conormal derivatives. Moreover, we use this to obtain uniqueness, existence and estimates for the solutions of the Robin-Problem. Here the main idea is to introduce a new Riemannian metric induced by the coefficients of the second order part of the elliptic operator. Then the operator takes a simpler form: Up to a relatively bounded perturbation of bound 0 , it coincides with a Laplace-Beltrami operator for the new metric. Regularity and perturbation theory for operator semigroups as in [9, Sect. 4] then yield the first part of the main theorem in its full generality. The second part follows from [17, Thm. 3.1] and [10, Thm. 1.1]. In the Appendix we collect some results about elliptic operators on manifolds with boundary.

In this paper the following notation is used. For a closed operator $T: D(T) \subset X \rightarrow$ $X$ on a Banach space $X$ we denote by $[D(T)]$ the Banach space $D(T)$ equipped with the graph norm $\|\bullet\|_{T}:=\|\bullet\|_{X}+\|T(\bullet)\|_{X}$ and indicate by $\hookrightarrow$ a continuous and by $\stackrel{c}{\hookrightarrow}$ a compact embedding. Moreover, we use Einstein's notation of sums, i.e.,

$$
x_{k} y^{k}:=\sum_{k=1}^{n} x_{k} y^{k}
$$

for $x:=\left(x_{1}, \ldots, x_{n}\right), y:=\left(y_{1}, \ldots, y_{n}\right)$. 


\section{The abstract setting}

The starting point of our investigation is the abstract setting proposed first in this form by [25] and successfully used, e.g., in [11,12,17] for the study of boundary perturbations.

\section{Abstract Setting 2.1 Consider}

(i) two Banach spaces $X$ and $\partial X$, called state and boundary space, respectively;

(ii) a densely defined maximal operator $A_{m}: D\left(A_{m}\right) \subset X \rightarrow X$;

(iii) a boundary (or trace) operator $L \in \mathcal{L}(X, \partial X)$;

(iv) a feedback operator $B: D(B) \subseteq X \rightarrow \partial X$.

Using these spaces and operators we define the operator $A^{B}: D\left(A^{B}\right) \subset X \rightarrow X$ with generalized Wentzell boundary conditions by

$$
A^{B} f:=A_{m} f, \quad D\left(A^{B}\right):=\left\{f \in D\left(A_{m}\right) \cap D(B): L A_{m} f=B f\right\} .
$$

For our purpose we need some more operators.

Notation 2.2 We denote the (closed) kernel of $L$ by $X_{0}:=\operatorname{ker}(L)$ and consider the restriction $A_{0}$ of $A_{m}$ given by

$$
A_{0}: D\left(A_{0}\right) \subset X \rightarrow X, \quad D\left(A_{0}\right):=\left\{f \in D\left(A_{m}\right): L f=0\right\} .
$$

The abstract Dirichlet operator associated with $A_{m}$ is, if it exists,

$$
L_{0}^{A_{m}}:=\left(\left.L\right|_{\operatorname{ker}\left(A_{m}\right)}\right)^{-1}: \partial X \rightarrow \operatorname{ker}\left(A_{m}\right) \subseteq X,
$$

i.e., $L_{0}^{A_{m}} \varphi=f$ is equal to the solution of the abstract Dirichlet problem

$$
\left\{\begin{array}{l}
A_{m} f=0, \\
L f=\varphi .
\end{array}\right.
$$

If it is clear which operator $A_{m}$ is meant, we simply write $L_{0}$.

Moreover for $\lambda \in \mathbb{C}$ we define the abstract Robin operator associated with $\left(\lambda, A_{m}, B\right)$ by

$$
R_{\lambda}:=\left(\left.(B-\lambda L)\right|_{\operatorname{ker}\left(A_{m}\right)}\right)^{-1}: \partial X \rightarrow \operatorname{ker}\left(A_{m}\right) \cap D(B) \subseteq X,
$$

i.e., $R_{\lambda} \varphi=f$ is equal to the solution of the abstract Robin problem

$$
\left\{\begin{array}{l}
A_{m} f=0, \\
B f-\lambda L f=\varphi .
\end{array}\right.
$$

If it is clear which operators $A_{m}$ and $B$ are meant, we simply write $R_{\lambda}$. 
Furthermore, we introduce the abstract Dirichlet-to-Neumann operator associated with $\left(A_{m}, B\right)$ defined by

$$
N^{A_{m}, B} \varphi:=B L_{0}^{A_{m}} \varphi, \quad D\left(N^{A_{m}, B}\right):=\left\{\varphi \in \partial X: L_{0}^{A_{m}} \varphi \in D(B)\right\} .
$$

If it is clear which operators $A_{m}$ and $B$ are meant, we call $N$ simply the (abstract) Dirichlet-to-Neumann operator. This Dirichlet-to-Neumann operator is an abstract version of the operators studied in many places, e.g., [19], [36, Sect. 7.11] and [35, Sect. II.5.1].

The Dirichlet-to-Neumann and the Robin operator are connected in the following way.

Lemma 2.3 If $L_{0}$ exists, we have $\lambda \in \rho(N)$ if and only if $R_{\lambda} \in \mathcal{L}(\partial X, X)$ exists. If one of these conditions is satisfied, we obtain

$$
R_{\lambda}=-L_{0} R(\lambda, N)
$$

Proof Assume that $R_{\lambda} \in \mathcal{L}(\partial X, X)$ exists. By the definition of $N$ the equation

$$
\lambda \varphi-N \varphi=\psi
$$

for $\varphi \in D(N), \psi \in \partial X$ is equivalent to

$$
\lambda L L_{0} \varphi-B L_{0} \varphi=\psi
$$

for $\varphi \in D(N), \psi \in \partial X$. This again is equivalent to

$$
-R_{\lambda} \psi=L_{0} \varphi
$$

Therefore, we have for $\varphi \in D(N), \psi \in \partial X$ the equivalence

$$
\mu \varphi-N \varphi=\psi \quad \Longleftrightarrow \quad R_{\lambda} \psi=-L_{0} \varphi
$$

Since $R_{\lambda}: \partial X \rightarrow \operatorname{ker}\left(A_{m}\right) \cap D(B)$ exists and $L_{0}: \partial X \rightarrow \operatorname{ker}\left(A_{m}\right)$ is an isomorphism, there exists a unique $\varphi \in D(N)$ for every $\psi \in \partial X$. Moreover its given by $\varphi=-L R_{\lambda, \mu} \psi$ and therefore the boundedness of the inverse follows from the boundedness of $L$ and $R_{\lambda}$. The formula for the resolvent of $N$ follows, since $\left.L\right|_{\operatorname{ker}\left(A_{m}\right)}$ is an isomorphism with inverse $L_{0}$ and the image of $R_{\lambda}$ is contained in $\operatorname{ker}\left(A_{m}\right)$.

Conversely, we assume that $\lambda \in \rho(N)$. Then (2.5) has a unique solution $\varphi \in D(N)$ for every $\psi \in \partial X$. Considering $f:=-L_{0} \varphi$ we obtain a unique solution of (2.3) and hence $R_{\lambda}$ exists. Boundedness follows from $R_{\lambda}=-L_{0} R(\lambda, N)$.

\section{Boundary problems for the Laplace-Beltrami operator}

In order to obtain a concrete realization of the above abstract objects we consider a smooth, compact, orientable Riemannian manifold $(\bar{M}, g)$ with smooth boundary 
$\partial M$, where $g$ denotes the Riemannian metric. Moreover, we take the Banach spaces $X:=\mathrm{C}(\bar{M})$ and $\partial X=\mathrm{C}(\partial M)$ and as the maximal operator the Laplace-Beltrami operator

$$
A_{m} f:=\Delta_{M}^{g} f, \quad D\left(A_{m}\right):=\left\{f \in \bigcap_{p>1} \mathrm{~W}_{\mathrm{loc}}^{2, p}(M) \cap \mathrm{C}(\bar{M}): \Delta_{M}^{g} f \in \mathrm{C}(\bar{M})\right\} .
$$

As feedback operator we take the normal derivative

$$
B f:=-g\left(\nabla_{M}^{g} f, \nu_{g}\right), \quad D(B):=\left\{f \in \bigcap_{p>1} \mathrm{~W}_{\mathrm{loc}}^{2, p}(M) \cap \mathrm{C}(\bar{M}): B f \in \mathrm{C}(\partial M)\right\},
$$

where $\nabla_{M}^{g}$ denotes the gradient on $M$, which in local coordinates is given as

$$
\left(\nabla_{M}^{g} f\right)^{l}=g^{k l} \partial_{k} f
$$

for $f \in \bigcap_{p>1} W^{1, p}(M)$. Moreover, $v_{g}$ is the outer normal on $\partial M$ given in local coordinates by

$$
v_{g}^{l}=g^{k l} v_{k}
$$

Furthermore, we choose $L$ as the trace operator, i.e.,

$$
L: X \rightarrow \partial X,\left.f \mapsto f\right|_{\partial M}
$$

which is bounded with respect to the supremum norm. Later on we will also need the unique bounded extension of $L$ to $\mathrm{W}^{1,2}(M)$, denoted by $\bar{L}: \mathrm{W}^{1,2}(M) \rightarrow \mathrm{L}^{2}(\partial M)$, and call it the (generalized) trace operator.

\subsection{The Laplace-Beltrami operator with Robin boundary conditions}

In this setting we consider the Laplace-Beltrami operator with Robin boundary conditions and prove existence, uniqueness and regularity for the solution of (2.3). Moreover, we show that this solution satisfies a maximum principle.

For this purpose we need the concept of a weak solution of (2.3). If $f \in D\left(A_{m}\right) \cap$ $D(B)$ is a solution of (2.3) we obtain by Green's Identity

$$
\int_{M} g\left(\nabla_{M}^{g} f, \nabla_{M}^{g} \bar{\phi}\right) \mathrm{dvol}_{M}^{g}=-\int_{\partial M} B f \bar{L} \phi \mathrm{dvol}_{\partial M}^{g}=-\int_{\partial M} \lambda \bar{L} f \bar{L} \phi \mathrm{dvol}_{\partial M}^{g}-\int_{\partial M} \varphi \bar{L} \phi \mathrm{dvol}_{\partial M}^{g}
$$

for all $\phi \in \mathrm{W}^{1,2}(M)$. This motivates the following definition.

Definition 3.1 (Weak solution of the Robin Problem) We call $f \in \mathrm{W}^{1,2}(M)$ a weak solution of (2.3) if it satisfies

$$
\mathfrak{a}(f, \phi):=\int_{M} g\left(\nabla_{M}^{g} f, \nabla_{M}^{g} \bar{\phi}\right) \mathrm{dvol}_{M}^{g}+\int_{\partial M} \lambda \bar{L} f \bar{L} \phi \mathrm{dvol}_{\partial M}^{g}=-\int_{\partial M} \varphi \bar{L} \phi \mathrm{dvol}_{\partial M}^{g}=: F(\phi)
$$


for all $\phi \in \mathrm{W}^{1,2}(M)$.

Definition 3.2 We call $f \in D\left(A_{m}\right) \cap D(B)$ a strong solution of (2.3) if it satisfies (2.3).

Next we prove the existence of such weak solutions.

Lemma 3.3 (Existence and Uniqueness of the weak solution of the Robin problem) For each $\operatorname{Re}(\lambda)>0$ and each $\varphi \in \mathrm{W}^{\frac{1}{2}, 2}(\partial M)$ the problem (2.3) has a unique weak solution.

Proof We consider $\mathfrak{a}$ and $F$ as defined above. Obviously $\mathfrak{a}$ is sesquilinear and $F$ is linear. By the Cauchy-Schwarz Inequality we have for $f, \phi \in \mathrm{W}^{1,2}(M)$ that

$$
|\mathfrak{a}(f, \phi)| \leq\left\|\nabla_{M}^{g} f\right\|_{\mathrm{L}^{2}(M)}\left\|\nabla_{M}^{g} \phi\right\|_{\mathrm{L}^{2}(M)}+|\lambda|\|\bar{L} f\|_{\mathrm{L}^{2}(\partial M)}\|\bar{L} \phi\|_{\mathrm{L}^{2}(\partial M)} \leq C\|f\|_{\mathrm{W}^{1,2}(M)}\|\phi\|_{\mathrm{W}^{1,2}(M)},
$$

hence $\mathfrak{a}: \mathrm{W}^{1,2}(M) \times \mathrm{W}^{1,2}(M) \rightarrow \mathbb{C}$ is bounded. Next we show that $\mathfrak{a}$ is coercive. If not, there exists a sequence $\left(u_{k}\right)_{k \in \mathbb{N}} \subset \mathrm{W}^{1,2}(M)$ such that

$$
\left\|u_{k}\right\|_{\mathrm{W}^{1,2}(M)}^{2}>k \operatorname{Re}\left(\mathfrak{a}\left(u_{k}, u_{k}\right)\right)
$$

for all $k \in \mathbb{N}$. We consider

$$
v_{k}:=\frac{u_{k}}{\left\|u_{k}\right\|_{\mathrm{W}^{1,2}(M)}} \in \mathrm{W}^{1,2}(M)
$$

and remark that $\left\|v_{k}\right\|_{\mathrm{W}^{1,2}(M)}=1$ and therefore

$$
\operatorname{Re}\left(\mathfrak{a}\left(v_{k}, v_{k}\right)\right)<\frac{1}{k}
$$

for all $k \in \mathbb{N}$. Since $\left(v_{k}\right)_{k \in \mathbb{N}}$ is bounded, by Rellich-Kondrachov (cf. [27, Cor. 3.7]) there exists a subsequence $\left(v_{k_{l}}\right)_{l \in \mathbb{N}}$ converging in $\mathrm{L}^{2}(M)$ to $v \in \mathrm{L}^{2}(M)$. On the other hand we have

$$
\left\|\nabla_{M}^{g} v_{k_{l}}\right\|_{L^{2}(M)} \leq \operatorname{Re}\left(\mathfrak{a}\left(v_{k_{l}}, v_{k_{l}}\right)\right)<\frac{1}{k_{l}}
$$

hence $\left(\nabla_{M}^{g} v_{k_{l}}\right)_{l \in \mathbb{N}}$ converges to 0 in $\mathrm{L}^{2}(M)$. This shows $v \in \mathrm{W}^{1,2}(M)$ and $\nabla_{M}^{g} v=0$. Moreover, we obtain

$$
\left\|\nabla_{M}^{g} v_{k_{l}}\right\|_{\mathrm{L}^{2}(M)}=\int_{M} g_{i j} g^{i r} g^{j s} \partial_{r} v_{k_{l}} \partial_{s} v_{k_{l}} \mathrm{dvol}_{M}^{g}=\int_{M} g^{r s} \partial_{r} v_{k_{l}} \partial_{s} v_{k_{l}} \mathrm{dvol}_{M}^{g}=\left\|\nabla v_{k_{l}}\right\|_{\mathrm{L}^{2}(M)},
$$

where $\nabla v_{k_{l}}$ denotes the covariant derivative of $v_{k_{l}}$. Therefore, $\left(v_{k_{l}}\right)_{l \in \mathbb{N}}$ converges in $\mathrm{W}^{1,2}(M)$ to $v$ with $\|v\|_{\mathrm{W}^{1,2}(M)}=1$. Moreover, we have

$$
\left\|\bar{L} v_{k_{l}}\right\|_{L^{2}(\partial M)}<\frac{1}{\operatorname{Re}(\lambda) k_{l}}
$$


and therefore

$$
\|\bar{L} v\|_{\mathrm{L}^{2}(\partial M)} \leq\left\|\bar{L} v-\bar{L} v_{k_{l}}\right\|_{\mathrm{L}^{2}(\partial M)}+\left\|\bar{L} v_{k_{l}}\right\|_{\mathrm{L}^{2}(\partial M)}<\frac{1}{\operatorname{Re}(\lambda) k_{l}}+C\left\|v-v_{k_{l}}\right\|_{\mathrm{W}^{1,2}(M)} \longrightarrow 0
$$

and hence $\bar{L} v=0$. Since $\nabla v=0$, we conclude $v=0$, which contradicts $\|v\|_{\mathrm{W}^{1,2}(M)}=$ 1. Hence, $\mathfrak{a}$ is coercive. Since

$$
|F(\phi)| \leq\|\varphi\|_{\mathrm{L}^{2}(\partial M)}\|\bar{L} \phi\|_{\mathrm{L}^{2}(\partial M)} \leq C\|\phi\|_{\mathrm{W}^{1,2}(\partial M)}
$$

for all $\phi \in \mathrm{W}^{1,2}(M)$ we conclude that $F: \mathrm{W}^{1,2}(M) \rightarrow \mathbb{C}$ is bounded. By the LaxMilgram and Fréchet-Riesz theorems it follows that $\mathfrak{a}(f, \phi)=F(\phi)$ for all $\phi \in$ $\mathrm{W}^{1,2}(M)$ has a unique solution $f \in \mathrm{W}^{1,2}(M)$.

Next we prove that every weak solution is even a strong solution.

Lemma 3.4 (Regularity of the Robin problem) If $\varphi \in \mathrm{C}(\partial M)$, every weak solution of (2.3) is a strong solution.

Proof By [36, Chap. 5., Prop. 1.6] we have $f \in \mathrm{C}^{2}(M) \subset \bigcap_{p>1} \mathrm{~W}_{\text {loc }}^{2, p}(M)$.

Therefore, we obtain by the fundamental lemma of the calculus of variation that $\Delta_{M}^{g} f=0$, in particular $\Delta_{M}^{g} f \in \mathrm{C}(\bar{M})$. Furthermore we have

$$
B f=\lambda L f+\varphi \in \mathrm{C}(\partial M) .
$$

Moreover we need a maximum principle for the Robin problem.

Lemma 3.5 A solution $f \in D\left(A_{m}\right) \cap D(B) \subset X$ of (2.3) satisfies the maximum principle

$$
|\operatorname{Re}(\lambda)| \cdot\|f\|_{X} \leq\|\varphi\|_{\partial X}
$$

for all $\operatorname{Re}(\lambda) \geq 0$ and $\varphi \in \partial X=\mathrm{C}(\partial M)$.

Proof We consider a point $p \in \bar{M}$, where $|f|$ and therefore $|f|^{2}$ assumes its maximum. By the interior maximum principle (cf. Theorem A.1) it follows that $q \in \partial M$. Hence, we have

$$
g(q)\left(\nabla_{M}^{g}|f|^{2}(q), v_{g}(q)\right) \geq 0
$$

From

$$
\begin{aligned}
g\left(\nabla_{M}^{g}|f|^{2}, v_{g}\right) & =g\left(\nabla_{M}^{g}(f \bar{f}), v_{g}\right)=2 \operatorname{Re} g\left(\left(\nabla_{M}^{g} f\right) \bar{f}, v_{g}\right)=2 \operatorname{Re}\left(g\left(\left(\nabla_{M}^{g} f\right), v_{g}\right) \bar{f}\right) \\
& =-2 \operatorname{Re}((B f) \bar{f})=-2 \operatorname{Re}(\varphi \bar{f})-2 \operatorname{Re}(\lambda)|f|^{2},
\end{aligned}
$$


we obtain

$$
\operatorname{Re}(\lambda)|f|^{2}(q) \leq-\operatorname{Re}(\varphi(q) \bar{f}(q)) \leq|\varphi|(q)|f|(q)
$$

Since $\operatorname{Re}(\lambda) \geq 0$, this implies

$$
|\operatorname{Re}(\lambda)| \cdot\|f\|_{X}=|\operatorname{Re}(\lambda)| \cdot|f|(p) \leq|\varphi|(p) \leq\|\varphi\|_{\partial X}
$$

Summing up we obtain the following.

Corollary 3.6 (Existence and Uniqueness of the solution of the Robin problem) For all $\operatorname{Re}(\lambda)>0$ and $\varphi \in \mathrm{C}(\partial M)$ the problem (2.3) has a unique solution.

Proof If $\varphi \in \mathrm{W}^{\frac{1}{2}, 2}(\partial M) \cap \mathrm{C}(\partial M)$ the claim follows by combining Lemmas 3.3 and 3.4 .

For general $\varphi \in \mathrm{C}(\partial M)$, the claim follows by density of $\mathrm{W}^{\frac{1}{2}, 2}(\partial M) \cap \mathrm{C}(\partial M) \subset$ $\mathrm{C}(\partial M)$ and the maximum principle Lemma 3.5.

\subsection{Generator property for the Dirichlet-to-Neumann operator}

Now we are able to prove our main result: The Dirichlet-to-Neumann operator generates a contractive and analytic semigroup of angle $\frac{\pi}{2}$ on $\partial X=\mathrm{C}(\partial M)$. To do so we represent the Dirichlet-to-Neumann operator as a relatively bounded perturbation of $W:=-\sqrt{-\Delta_{\partial M}^{g}}$.

We first need the existence of the associated Dirichlet operator.

Lemma 3.7 The Dirichlet operator $L_{0} \in \mathcal{L}(\partial X, X)$ exists.

Proof This follows by [36, Chap. 5. (2.26)], Theorem A.1 and Corollary A.4.

Next we prove a first generation result for the Dirichlet-to-Neumann operator.

Proposition 3.8 The Dirichlet-to-Neumann operator $N$ defined in (2.4) generates a contraction semigroup on $\partial X$.

Proof By elliptic regularity theory (cf. [36, Chap. 5.5. Ex. 2]), we have the inclusions

$$
L_{0} \mathrm{C}^{2}(\partial M) \subset \mathrm{C}^{1}(\bar{M}) \subset D(B) .
$$

Since $\mathrm{C}^{2}(\partial M)$ is dense in $\partial X, N$ is densely defined. By Lemma 2.3 and Corollary 3.6 it follows that the resolvent $R(\lambda, N)$ exists for all $\operatorname{Re}(\lambda)>0$. By the interior maximum principle $\left.L\right|_{\operatorname{ker}\left(A_{m}\right)}: \operatorname{ker}\left(A_{m}\right) \subset X \rightarrow \partial X$ is an isometry. Therefore, Lemmas 2.3 and 3.5 imply

$$
\|R(\lambda, N) \varphi\|_{\partial X} \leq \frac{1}{|\operatorname{Re}(\lambda)|}\|\varphi\|_{\partial X}
$$


for all $\operatorname{Re}(\lambda)>0$ and $\varphi \in \partial X$. Hence, the claim follows by the Hille-Yosida theorem (cf. [18, Thm. II.3.5]).

Now we prove the main result of this subsection.

Theorem 3.9 The Dirichlet-to-Neumann operator $N$ given by (2.4) for (3.1) and (3.2) generates an analytic semigroup of angle $\frac{\pi}{2}$ on $\partial X$.

We proceed as in the proof of [20, Thm. 2.1]. Let $\bar{N}$ and $\bar{W}$ be the closure of $N$ and $W$, respectively, in $Y:=\mathrm{L}^{2}(\partial M)$. Moreover we need results from the theory of pseudo differential operators. We use the notation from [35] and denote by $\operatorname{OPS}^{k}(\partial M)$ the pseudo differential operators of order $k \in \mathbb{Z}$ on $\partial M$.

Step 1 Then the part $\left.\bar{N}\right|_{\partial X}$ coincides with $N$.

Proof By Proposition 3.8 the Dirichlet-to-Neumann operator $N$ is densely defined and $\lambda-N$, considered as an operator on $Y$, has dense range $\operatorname{rg}(\lambda-N)=\partial X \subset Y$ for all $\lambda>0$. By Green's Identity we have

$$
\int_{M} g\left(\nabla_{M}^{g} f, \nabla_{M}^{g} f\right) d \operatorname{vol}_{M}+\int_{M} f \Delta_{M} f d \operatorname{vol}_{M}=\int_{\partial M} g\left(\nabla_{M}^{g} f, v_{g}\right) L f d \operatorname{vol}_{\partial M} .
$$

Hence, for $f:=L_{0}^{A_{m}} \varphi$ with $\varphi \in D(N)$ we obtain

$$
0 \leq \int_{M} g\left(\nabla_{M}^{g} f, \nabla_{M}^{g} f\right) d \operatorname{vol}_{M}=-\int_{\partial M} \varphi N \varphi d \operatorname{vol}_{\partial M}
$$

since $\Delta_{M}^{g} f=0$. Hence, $N$ as an operator on $Y$ is dissipative. By the Lumer-Phillips theorem (see [18, Thm. II.3.15]) the closure $\bar{N}$ of $N$ exists and generates a contraction semigroup on $Y$. This implies that on $\partial X$ we have

$$
\left.(1-N) \subseteq(1-\bar{N})\right|_{\partial X},
$$

where $1-N$ is surjective and $1-\bar{N}$ is injective on $\partial X$. This is possible only if the domains $D(1-N)$ and $D(1-\bar{N})$ coincide, i.e., $\left.\bar{N}\right|_{\partial X}=N$.

Step 2 The operator $W:=-\sqrt{-\Delta_{\partial M}^{g}}$ generates an analytic semigroup of angle $\frac{\pi}{2}$ on $\partial X$.

Proof By [10, Cor. 3.6] the Laplace-Beltrami operator $\Delta_{\partial M}^{g}$ generates an analytic semigroup of angle $\frac{\pi}{2}$ on $\mathrm{C}(\partial M)=\partial X$. Hence, the assertion follows by $[1$, Thm. 3.8.3].

Step 3 The operator $\bar{W}:=-\sqrt{-\overline{\Delta_{\partial M}^{g}}}$ satisfies $W=\left.\bar{W}\right|_{\partial X}$. 
Proof By [35, Chap. 8, Prop. 2.4] the space $C^{\infty}(\partial M)$ is a core for $\overline{\Delta_{\partial M}^{g}}$ and by [1, Prop. 3.8.2] the domain $D\left(\overline{\Delta_{\partial M}^{g}}\right)$ is a core for $\bar{W}$. Hence, $\mathrm{C}^{\infty}(\partial M)$ is a core for $\bar{W}$ and since $\mathrm{C}^{\infty}(\partial M) \subset D(W)$ we obtain that $D(W)$ is a core for $\bar{W}$ on $Y$. This implies that $\bar{W}$ is indeed the closure of $W$ in $Y$. Moreover, we obtain

$$
\left.(1-W) \subseteq(1-\bar{W})\right|_{\partial X},
$$

where $1-W$ is surjective and $1-\bar{W}$ is injective on $\partial X$. This is possible only if for the domains we have

$$
D(1-W)=D(1-\bar{W}),
$$

i.e., $\left.\bar{W}\right|_{\partial X}=W$.

Step 4 The domain of $W$ can be compactly embedded into the Hölder continuous functions, i.e., $[D(W)] \stackrel{c}{\hookrightarrow} \mathrm{C}^{\alpha}(M)$ for all $\alpha \in(0,1)$.

Proof Consider $\bar{R}:=(1+\bar{W})^{-1}$. Then, by [35, Chap. XII.1], $\bar{R} \in \mathrm{OPS}^{-1}(\partial M)$ and since $\varphi \in \partial X=\mathrm{C}(\partial M)$ we have by [35, Chap. XI, Thm. 2.5] that $\bar{R} \varphi \in \mathrm{W}^{1, p}(\partial M)$ for all $p>1$. Hence, $D(W)=\bar{R} \mathrm{C}(\partial M) \subset \mathrm{W}^{1, p}(\partial M)$. Moreover, by Sobolev embedding (see [2, Chap. V. and Rem. 5.5.2])

$$
\mathrm{W}^{1, p}(\partial M) \hookrightarrow \mathrm{C}(\partial M)
$$

for $p>n-1$, where $n:=\operatorname{dim}(\bar{M})$. By the closed graph theorem we obtain

$$
[D(W)] \hookrightarrow \mathrm{W}^{1, p}(\partial M)
$$

for $p>n-1$. Since Rellich's embedding (see [2, Thm. 6.2, Part III.]) implies

$$
\mathrm{W}^{1, p}(\partial M) \stackrel{c}{\hookrightarrow} \mathrm{C}^{\alpha}(\partial M)
$$

for $p>\frac{n-1}{1-\alpha}$, the claim follows.

Step 5 The difference $\bar{P}:=\bar{N}-\bar{W} \in \operatorname{OPS}^{0}(\partial M)$ is a pseudo differential operator of order 0 . Moreover, $\bar{P}$ considered as an operator on $Y$ is bounded.

Proof This follows from [36, App. C, (C.4)] and [35, Chap. XI, Thm. 2.2].

Step 6 The part $P:=\left.\bar{P}\right|_{\mathrm{C}^{\alpha}(\partial M)}: \mathrm{C}^{\alpha}(\partial M) \rightarrow \mathrm{C}^{\alpha}(\partial M)$ is bounded. Moreover, the operator $P$ considered on $\partial X$ is relatively $W$-bounded with bound 0 .

Proof Form [35, Chap. XI, Thm 2.2] it follows $P \in \mathcal{L}\left(\mathrm{C}^{\alpha}(\partial M)\right)$. By Step 4 we have

$$
[D(W)] \stackrel{c}{\hookrightarrow} \mathrm{C}^{\alpha}(\partial M) \hookrightarrow \mathrm{C}(\partial M) .
$$


Therefore, by Ehrling's lemma (cf. [34, Thm. 6.99]), for every $\varepsilon>0$ there exists a constant $C_{\varepsilon}>0$ such that

$$
\|\varphi\|_{C^{\alpha}(\partial M)} \leq \varepsilon\|\varphi\|_{W}+C_{\varepsilon}\|\varphi\|_{\infty}
$$

for every $\varphi \in D(W)$, i.e. $P$ is relatively $W$-bounded with bound 0 .

Step 7 (Proof of Theorem 3.9)

Proof First we note that by Step 5 we have

$$
\bar{N}=\bar{W}-\bar{P}
$$

and therefore using the Steps 1, 3, 6 it follows that

$$
N=\left.\bar{N}\right|_{\partial X}=\left.\left.(\bar{W}-\bar{P})\right|_{\partial X} \supseteq \bar{W}\right|_{\partial X}-P=W-P .
$$

On the other hand, by Steps 2, 6 and [18, Lem. III.2.6], $W-P$ generates an analytic semigroup of angle $\frac{\pi}{2}$ on $\partial X$. Moreover, $\lambda \in \rho(N) \cap \rho(W-P)$ for $\lambda$ large enough. This implies equality in (3.4) and hence the claim.

Remark 1 After we finished this paper, a different proof of Theorem 3.9 came to our mind, based on the work of ter Elst and Ouhabaz [15].

First, note that by the remark at the end of [15, Sect. 1] all results in [15] still be true on Riemannian manifolds. Applying the same arguments as in the proof of [16, Prop. 2.3], using [15, Thm. 2.6] instead of [16, Thm. 2.1], we get that the Dirichletto-Neumann operator generates a strongly continuous semigroup on $\mathrm{C}(\partial M)$. Using [15, Cor. 5. 14] one obtains in the same way as in the proof of [16, Prop. 3.3] that the Dirichlet-to-Neumann operator generates a holomorphic semigroup of angle $\frac{\pi}{2}$ on $\mathrm{C}(\partial M)$. Combining these two results implies that the Dirichlet-to-Neumann operator generates an analytic semigroup of angle $\frac{\pi}{2}$ on $\mathrm{C}(\partial M)$.

Corollary 3.10 The Dirichlet-to-Neumann operator generates a compact semigroup on $\mathrm{C}(\partial M)$.

Proof By (3.3) the operator $W$ has compact resolvent. Since the Dirichlet-to-Neumann operator $N$ and $W$ differ only by a relatively bounded perturbation of bound 0 , it has compact resolvent by [18, III.-(2.5)]. Hence the claim follows by Theorem 3.9 and [18, Thm. II.4.29].

Remark 2 We can insert a strictly positive function $0<\beta \in \mathrm{C}(\partial M)$ and consider $\tilde{B}:=\beta \cdot B$. Then by multiplicative perturbation theory (cf. [31, Sect. III.1]) the same generation result as above holds true.

\subsection{The Laplace-Beltrami operator with Wentzell boundary conditions}

In this subsection we study the Laplace-Beltrami operator with Wentzell boundary conditions and prove that it generates an analytic semigroup of angle $\frac{\pi}{2}$ on $X=\mathrm{C}(\bar{M})$. To show this, we verify the assumptions of [9, Thm. 3.1]. 
Lemma 3.11 The feedback operator $B$ is relatively $A_{0}$-bounded with bound 0 .

Proof By [36, Chap. 5., Thm. 1.3] and the closed graph theorem we obtain

$$
\left[D\left(A_{0}\right)\right] \hookrightarrow \mathrm{W}^{2, p}(M)
$$

Rellich's embedding (see [2, Thm. 6.2, Part III.]) implies

$$
\mathrm{W}^{2, p}(M) \stackrel{c}{\hookrightarrow} \mathrm{C}^{1, \alpha}(M) \stackrel{c}{\hookrightarrow} \mathrm{C}^{1}(\bar{M})
$$

for $p>\frac{n-1}{1-\alpha}$, where $n:=\operatorname{dim}(\bar{M})$, so we obtain

$$
\left[D\left(A_{0}\right)\right] \stackrel{c}{\hookrightarrow} \mathrm{C}^{1}(\bar{M}) \hookrightarrow \mathrm{C}(\bar{M}) .
$$

Therefore, by Ehrling's lemma (cf. [34, Thm. 6.99]), for every $\varepsilon>0$ there exists a constant $C_{\varepsilon}>0$ such that

$$
\|f\|_{C^{1}(\bar{M})} \leq \varepsilon\|f\|_{A_{0}}+C_{\varepsilon}\|f\|_{X}
$$

for every $f \in D\left(A_{0}\right)$. Since $B \in \mathcal{L}\left(\mathrm{C}^{1}(\bar{M}), \partial X\right)$, this implies the claim.

Now we prove the generator result for the operator with Wentzell boundary conditions.

Theorem 3.12 The operator $A^{B}$ with Wentzell boundary conditions given by (2.1) for (3.1) and (3.2) generates a compact and analytic semigroup of angle $\frac{\pi}{2}$ on $X=\mathrm{C}(\bar{M})$.

Proof We verify the assumptions from [17, Thm. 3.1]. The operator $A_{0}$ with Dirichlet boundary conditions is sectorial of angle $\frac{\pi}{2}$ with compact resolvent by [10, Thm. 2.8] and [10, Cor. 3.4]. Moreover the Dirichlet operator $L_{0}$ exists by Lemma 3.7 and the feedback operator $B$ is relatively $A_{0}$-bounded of bound 0 by Lemma 3.11. Lastly, the Dirichlet-to-Neumann operator $N$ generates a compact and analytic semigroup of angle $\frac{\pi}{2}$ on $\mathrm{C}(\partial M)$ by Theorem 3.9 and Corollary 3.10. Now the claim follows from [17, Thm. 3.1].

Remark 3 As in Remark 2 we can insert a strictly positive, continuous function $\beta>0$ and the same result as Theorem 3.12 becomes true.

\section{Strictly elliptic operators on continuous functions on a compact manifold with boundary}

In this section we consider strictly elliptic second-order differential operators with generalized Wentzell boundary conditions on $X:=\mathrm{C}(\bar{M})$ for a smooth, compact, 
orientiable, Riemannian manifold $(\bar{M}, g)$ with smooth boundary $\partial M$. To this end, we take real-valued functions

$$
a_{j}^{k}=a_{k}^{j} \in \mathrm{C}^{\infty}(\bar{M}), \quad b_{j} \in \mathrm{C}_{c}(M), \quad c \in \mathrm{C}(\bar{M}), d \in \mathrm{C}(\partial M), \quad 1 \leq j, k \leq n,
$$

satisfying the strict ellipticity condition

$$
a_{j}^{k}(q) g^{j l}(q) X_{k}(q) X_{l}(q)>0
$$

for all co-vectorfields $X_{k}, X_{l}$ on $\bar{M}$ with $\left(X_{1}(q), \ldots, X_{n}(q)\right) \neq(0, \ldots, 0)$. Further, denote $|a|:=\operatorname{det}\left(a_{j}^{k}\right)$. Then we define the maximal operator in divergence form as

$$
\begin{aligned}
\tilde{A}_{m} f & :=\sqrt{|a|} \operatorname{div}_{g}\left(\frac{1}{\sqrt{|a|}} a \nabla_{M}^{g} f\right)+\left\langle b, \nabla_{M}^{g} f\right\rangle+c f, \\
D\left(\tilde{A}_{m}\right) & :=\left\{f \in \bigcap_{p>1} \mathrm{~W}_{\text {loc }}^{2, p}(M) \cap \mathrm{C}(\bar{M}): \tilde{A}_{m} f \in \mathrm{C}(\bar{M})\right\} .
\end{aligned}
$$

As feedback operator we take

$\tilde{B} f:=-g\left(a \nabla_{M}^{g} f, v_{g}\right)+d L f, \quad D(\tilde{B}):=\left\{f \in \bigcap_{p>1} \mathrm{~W}_{\mathrm{loc}}^{2, p}(M) \cap \mathrm{C}(\bar{M}): \tilde{B} f \in \mathrm{C}(\partial M)\right\}$.

Corresponding to $L$ we choose $\partial X:=\mathrm{C}(\partial M)$.

The key idea is to reduce the strictly elliptic operator and the conormal derivative on $\bar{M}$, equipped by $g$, to the Laplace-Beltrami operator and to the normal derivative on $\bar{M}$, endowed by a new metric $\tilde{g}$.

For this purpose we consider a $(2,0)$-tensorfield on $\bar{M}$ given by

$$
\tilde{g}^{k l}=a_{i}^{k} g^{i l}
$$

Its inverse $\tilde{g}$ is a $(0,2)$-tensorfield on $\bar{M}$, which is a Riemannian metric since $a_{j}^{k} g^{j l}$ is strictly elliptic on $\bar{M}$. We denote $\bar{M}$ with the old metric by $(\bar{M}, g)$ and with the new metric by $(\bar{M}, \tilde{g})$ and remark that $(\bar{M}, \tilde{g})$ is a smooth, compact, orientable Riemannian manifold with smooth boundary $\partial M$. Since the differentiable structures of $(\bar{M}, g)$ and $(\bar{M}, \tilde{g})$ coincide, the identity

$$
\mathrm{Id}:(\bar{M}, g) \longrightarrow(\bar{M}, \tilde{g})
$$

is a $C^{\infty}$-diffeomorphism. Hence, the spaces

$$
\begin{aligned}
X & :=\mathrm{C}(\bar{M}):=\mathrm{C}((\bar{M}, \tilde{g}))=\mathrm{C}((\bar{M}, g)) \\
\text { and } \quad \partial X & :=\mathrm{C}(\partial M):=\mathrm{C}((\partial M, \tilde{g}))=\mathrm{C}((\partial M, g))
\end{aligned}
$$


coincide. Moreover, [28, Prop. 2.2] implies that the spaces

$$
\begin{aligned}
\mathrm{L}^{p}(M) & :=\mathrm{L}^{p}((M, \tilde{g}))=\mathrm{L}^{p}((M, g)), \\
\mathrm{W}^{k, p}(M) & :=\mathrm{W}^{k, p}((M, \tilde{g}))=\mathrm{W}^{k, p}((M, g)), \\
\mathrm{L}_{\mathrm{loc}}^{p}(M) & :=\mathrm{L}_{\mathrm{loc}}^{p}((M, \tilde{g}))=\mathrm{L}_{\mathrm{loc}}^{p}((M, g)), \\
\mathrm{W}_{\mathrm{loc}}^{k, p}(M) & :=\mathrm{W}_{\mathrm{loc}}^{k, p}((M, \tilde{g}))=\mathrm{W}_{\mathrm{loc}}^{k, p}((M, g))
\end{aligned}
$$

for all $p>1$ and $k \in \mathbb{N}$ coincide. We now denote by $A_{m}$ and $B$ the operators defined as in Sect. 3 with respect to $\tilde{g}$. Moreover we denote $\hat{A}_{m}$ the operator defined in (4.3) for $b_{k}=c=0$.

\subsection{The associated Dirichlet-to-Neumann operator and the Robin problem}

In this subsection we study the Dirichlet-to-Neumann operator $N^{\tilde{A}_{m}, \tilde{B}}$ associated with $\tilde{A}_{m}$ and $\tilde{B}$. First we prove that the generator properties of the Dirichlet-to-Neumann operators associated with $\left(\tilde{A}_{m}, \tilde{B}\right)$ and $\left(A_{m}, B\right)$ are closely related.

Lemma 4.1 The operators $\hat{A}_{m}$ and $\tilde{A}_{m}$ differ only by a relatively $A_{m}$-bounded perturbation of bound 0 .

Proof From (4.5) we define

$$
P_{1} f:=b_{l} g^{k l} \partial_{k} f
$$

for $f \in D\left(A_{m}\right) \cup D\left(\hat{A}_{m}\right)$. Morreys embedding (cf. [2, Chap. V. and Rem. 5.5.2]) implies

$$
\left[D\left(\hat{A}_{m}\right)\right] \stackrel{c}{\hookrightarrow} \mathrm{C}^{1}(M) \hookrightarrow \mathrm{C}(M)
$$

Since $b_{l} \in \mathrm{C}_{c}(M)$ we obtain

$$
\begin{aligned}
\left\|P_{1} f\right\|_{\mathrm{C}(\bar{M})} & \leq \sup _{q \in \bar{M}}\left|b_{l}(q) g^{k l}(q)\left(\partial_{k} f\right)(q)\right| \\
& =\sup _{q \in M}\left|b_{l}(q) g^{k l}(q)\left(\partial_{k} f\right)(q)\right| \\
& \leq C \sum_{k=1}^{n}\left\|\partial_{k} f\right\|_{\mathrm{C}(M)}
\end{aligned}
$$

and therefore $P_{1} \in \mathcal{L}\left(\mathrm{C}^{1}(M), \mathrm{C}(\bar{M})\right)$. Hence $D\left(\hat{A}_{m}\right) \subset D\left(\tilde{A}_{m}\right)$. Further $D\left(\hat{A}_{m}\right) \supset$ $D\left(\tilde{A}_{m}\right)$ follows from $\hat{A}_{m} f=\tilde{A}_{m} f-P f$. By (4.6) we conclude from Ehrling's Lemma (see [34, Thm. 6.99]) that

$$
\begin{aligned}
\left\|P_{1} f\right\|_{\mathrm{C}(\bar{M})} \leq C\|f\|_{\mathrm{C}^{1}(M)} & \leq \varepsilon\left\|\hat{A}_{m} f\right\|_{\mathrm{C}(\bar{M})}+\varepsilon\|f\|_{\mathrm{C}(\bar{M})}+C(\varepsilon)\|f\|_{\mathrm{C}(M)} \\
& \leq \varepsilon\left\|\hat{A}_{m} f\right\|_{\mathrm{C}(\bar{M})}+\tilde{C}(\varepsilon)\|f\|_{\mathrm{C}(\bar{M})}
\end{aligned}
$$


for $f \in D\left(\hat{A}_{m}\right)$ and all $\varepsilon>0$ and hence $P_{1}$ is relatively $A_{m}$-bounded of bound 0 . Finally, remark that

$$
P_{2} f:=c \cdot f, \quad D\left(P_{2}\right):=\mathrm{C}(\bar{M})
$$

is bounded and that

$$
\tilde{A}_{m} f=\hat{A}_{m} f+P_{1} f+P_{2} f
$$

for $f \in D\left(\hat{A}_{m}\right)$.

Lemma 4.2 The operator $\hat{A}_{m}$ equals to the Laplace-Beltrami operator $\Delta_{m}^{\tilde{g}}$.

Proof We have to verify this equality for all $q \in \bar{M}$. Choose local coordinates around $q \in \bar{M}$ and calculate

$$
\begin{aligned}
\hat{A}_{m} f & =\frac{1}{\sqrt{|g|}} \sqrt{|a|} \partial_{j}\left(\sqrt{|g|} \frac{1}{\sqrt{|a|}} a_{l}^{j} g^{k l} \partial_{k} f\right) \\
& =\frac{1}{\sqrt{|\tilde{g}|}} \partial_{j}\left(\sqrt{|\tilde{g}|} \tilde{g}^{k l} \partial_{k} f\right)=\Delta_{m}^{\tilde{g}} f
\end{aligned}
$$

for $f \in D\left(\hat{A}_{m}\right)=D\left(\Delta_{m}^{\tilde{g}}\right)$, since $|g|=|a| \cdot|\tilde{g}|$.

Lemma 4.3 The operators $B$ and $\tilde{B}$ differ only by a bounded perturbation.

Proof Note that the Sobolev spaces coincide. We have to verify this equality for all $q \in \bar{M}$. Choose local coordinates around $q \in \bar{M}$ and compute

$$
\begin{aligned}
\tilde{B} f & =-g_{i j} g^{j l} a_{l}^{k} \partial_{k} f g^{i m} v_{m}+d L f \\
& =-g_{i j} \tilde{g}^{j l} \partial_{k} f g^{i m} v_{m}+b_{0} L f \\
& =-\tilde{g}_{i j} \tilde{g}^{j l} \partial_{k} f \tilde{g}^{i m} v_{m}+d L f \\
& =B f+d L f
\end{aligned}
$$

for $f \in D(B)$. Since $d \cdot L f \in \mathrm{C}(\partial M)$ we obtain $D(B)=D(\tilde{B})$ and $B$ and $\tilde{B}$ differ only by the bounded perturbation $d \cdot L$.

Lemma 4.4 The Dirichlet-to-Neumann operator $N^{\tilde{A}_{m}, \tilde{B}}$ associated with $\tilde{A}_{m}$ and $\tilde{B}$ generates a compact and analytic semigroup of angle $\alpha>0$ on $\partial X$ if and only if $N^{A_{m}, B}$ associated with $A_{m}$ and $B$ does so.

Proof Let $P$ be the perturbation defined in the proof of Lemma 4.1. By Lemma 4.1 $P$ is relatively $A_{m}$-bounded of bound 0 . Moreover, $\tilde{B}$ and $B$ only differ by a bounded perturbation by Lemma 4.3. Hence, the claim follows by [9, Prop. 4.7].

Theorem 4.5 The Dirichlet-to-Neumann operator $N^{\tilde{A}_{m}, \tilde{B}}$ given by (2.4) for (4.3) and (4.4) generates a compact and analytic semigroup of angle $\frac{\pi}{2}$ on $X=C(\partial M)$. 
Proof The claim follows by Theorem 3.9 and Lemma 4.4 .

Remark 4 As in Remark 2 we can insert a strictly positive, continuous function $\beta>0$ and the same result as Theorem 3.9 becomes true.

Remark 5 Theorem 4.5 improves and generalizes the main result in [19]. If we consider $M=\Omega \subset \mathbb{R}^{n}$ equipped with the euclidean metric $g=\delta$, we obtain the maximal angle $\frac{\pi}{2}$ of analyticity in this case. This is the main result in [16] for smooth coefficients.

Now we use Theorem 4.5 to obtain existence and uniqueness for the associated Robin problem (2.3). Moreover, we obtain a maximum principle for the solutions of these problems.

Corollary 4.6 (Existence, uniqueness and maximum principle for the general Robin problem) There exists $\omega \in \mathbb{R}$ such that for all $\lambda \in \mathbb{C} \backslash(-\infty, \omega)$ the problem (2.3) has a unique solution $u \in D\left(A_{m}\right) \cap D(B)$. This solution satisfies the maximum principle

$$
|\lambda| \max _{p \in \bar{M}}|u(p)| \leq C|\lambda| \max _{p \in \partial M}|u(p)|=C|\lambda|\|L u\|_{\partial X} \leq \tilde{C}\|\varphi\|_{\partial X}=\tilde{C} \max _{p \in \partial M}|\varphi(p)| .
$$

Proof The existence and uniqueness follows immediately by Theorem 4.5 . The first inequality is the interior maximum principle. The second inequality is a direct consequence from Lemma 2.3 and Theorem 4.5.

\subsection{The associated operator $\tilde{A}^{\tilde{B}}$ with Wentzell boundary conditions}

Lemma 4.7 The operator $\tilde{A}^{\tilde{B}}$ generates a compact and analytic semigroup of angle $\alpha>0$ on $X$ if and only if $A^{B}$ does.

Proof As seen in the proof of Lemma 4.4, the operators $A_{m}$ and $\tilde{A}_{m}$ differ only by a relatively $A_{m}$-bounded perturbation with bound 0 while $B$ and $\tilde{B}$ differ only by a bounded perturbation. Therefore, the claim follows by [9, Thm. 4.2].

Theorem 4.8 The operator $\tilde{A}^{\tilde{B}}$ given by (2.1) for (4.3) and (4.4) generates a compact and analytic semigroup of angle $\frac{\pi}{2}$ on $X=\mathrm{C}(\bar{M})$.

Proof The claim follows by Theorem 3.12 and Lemma 4.7.

Remark 6 As in Remark 2 we can insert a strictly positive, continuous function $\beta>0$ and the same result as Theorem 4.8 becomes true.

Remark 7 Theorem 4.8 improves and generalizes [17, Cor. 4.5]. If we consider $M=$ $\Omega \subset \mathbb{R}^{n}$ equipped with the euclidean metric $g=\delta$, we obtain the maximal angle $\frac{\pi}{2}$ of analyticity. 
Corollary 4.9 By Theorem 4.8 the initial boundary problem

$$
\begin{cases}\frac{d}{d t} u(t, p)=\sqrt{|a(p)|} \operatorname{div}_{g}\left(\frac{1}{\sqrt{|a(p)|}} a(p) \nabla_{M}^{g} u(t, p)\right) & \\ +\left\langle b(p), \nabla_{M}^{g} u(t, p)\right\rangle+c(p) u(t, p) & \text { for } t \geq 0, p \in \bar{M}, \\ \frac{d}{d t} u(t, p)=-\beta g\left(a(p) \nabla_{M}^{g} u(t, p), v_{g}(p)\right)+d(p) u(t, p) & \text { for } t \geq 0, p \in \partial M, \\ u(0, p)=u_{0}(p) & \text { for } p \in \bar{M}\end{cases}
$$

for $a, b, c, d$ as in (4.1), $\beta>0$ and $u_{0}(p) \in D\left(A^{B}\right)$ has a unique solution on $\mathrm{C}(\bar{M})$. This solution is governed by an analytic semigroup in the right half-plane.

Finally, we consider the elliptic problem

$$
\left\{\begin{array}{l}
A_{m} f-\lambda f=h \\
L A_{m} f=B f,
\end{array}\right.
$$

for $f \in D\left(A_{m}\right) \cap D(B)$ and $h \in X=\mathrm{C}(\bar{M})$. Then the following holds.

Corollary 4.10 There exists $\omega \in \mathbb{R}$ such that for all $\lambda \in \mathbb{C} \backslash(-\infty, \omega)$ the problem (4.7) has a unique solution $u \in D\left(A_{m}\right) \cap D(B)$. This solution satisfies the maximum principle

$$
|\lambda| \max _{p \in \bar{M}}|f(p)|=|\lambda|\|f\|_{X} \leq C\|h\|_{X}=C \max _{p \in \bar{M}}|h(p)| .
$$

Proof This follows immediately by Theorem 4.8 .

Acknowledgements The author wishes to thank Professor Simon Brendle and Professor Klaus-Jochen Engel for many helpful suggestions and discussions. Further the author wishes to thank Professor Tom ter Elst for many helpful comments; in particular for an explanation of Remark 1.

Funding Open Access funding enabled and organized by Projekt DEAL.

Open Access This article is licensed under a Creative Commons Attribution 4.0 International License, which permits use, sharing, adaptation, distribution and reproduction in any medium or format, as long as you give appropriate credit to the original author(s) and the source, provide a link to the Creative Commons licence, and indicate if changes were made. The images or other third party material in this article are included in the article's Creative Commons licence, unless indicated otherwise in a credit line to the material. If material is not included in the article's Creative Commons licence and your intended use is not permitted by statutory regulation or exceeds the permitted use, you will need to obtain permission directly from the copyright holder. To view a copy of this licence, visit http://creativecommons.org/licenses/by/4.0/.

\section{Appendix A}

In the appendix we collect some results about elliptic differential equations. Even if the proofs follow by standard localization arguments from the analogous results on bounded domains we will give it here for the sake of completeness and convenience of the reader. 
Consider a smooth, compact, Riemannian manifold $(\bar{M}, g)$ with smooth boundary $\partial M$ of dimension $n$ and a strictly elliptic second order differential operator. To this end, we take real-valued functions

$$
a_{j}^{k}=a_{k}^{j} \in \mathrm{C}^{\infty}(\bar{M}), \quad b_{j} \in \mathrm{C}_{c}(M), \quad c \in \mathrm{C}(\bar{M}), d \in \mathrm{C}(\partial M), \quad 1 \leq j, k \leq n,
$$

satisfying the strict ellipticity condition

$$
a_{j}^{k}(q) g^{j l}(q) X_{k}(q) X_{l}(q)>0
$$

for all co-vectorfields $X_{k}, X_{l}$ on $\bar{M}$ with $\left(X_{1}(q), \ldots, X_{n}(q)\right) \neq(0, \ldots, 0)$. Further, denote $|a|:=\operatorname{det}\left(a_{j}^{k}\right)$. Then we define the formal operator in divergence form as

$$
A_{m}:=\sqrt{|a|} \operatorname{div}_{g}\left(\frac{1}{\sqrt{|a|}} a \nabla_{M}^{g}\right)+\left\langle b, \nabla_{M}^{g}\right\rangle+c .
$$

Consider the inequality

$$
A_{m} f \geq h \text { on } \bar{M} \text {. }
$$

Since $\bar{M}$ is compact, there exists a finite atlas $\left(U_{i}, \phi_{i}\right)$ with $i=1, \ldots, r$, i.e. $\bar{M}=$ $\bigcup_{i=1}^{r} U_{i}$ and $\phi_{i}: U_{i} \rightarrow V_{i} \subset \mathbb{R}_{+}^{n}$ are diffeomorphisms. Let $\left(\chi_{i}\right)_{i=1, \ldots, r}$ be the partition of unity subordinated to the covering $\left(U_{i}\right)_{i \in 1, \ldots, r}$, i.e. $\chi_{i} \in \mathrm{C}_{c}^{\infty}\left(U_{i}, \mathbb{R}\right), \chi_{i}(q) \in[0,1]$ and $\sum_{i=1}^{r} \chi_{i}=1$. Denote by $f_{i}:=\chi_{i} \cdot f, h_{i}:=\chi_{i} \cdot h, \tilde{f}_{i}:=f_{i} \circ \phi_{i}^{-1}, \tilde{h}_{i}=h_{i} \circ \phi_{i}^{-1}$. Further let

$$
A_{i}:=\sqrt{\frac{|a|}{|g|}} \partial_{i}\left(\sqrt{\frac{|g|}{|a|}} g^{k l} a_{k}^{i} \partial_{l}\right)+b^{k} \partial_{k}+c .
$$

Since by definition $A f=\sum_{i=1}^{r} A_{i} f_{i}$, we obtain

$$
A_{i} f_{i} \geq h_{i} \text { on } U_{i}
$$

for all $i \in\{1, \ldots, r\}$. Using

$$
\begin{aligned}
& \tilde{a}^{i j}:=\left(g^{i k} a_{k}^{j} \partial_{i} \chi \partial_{j} \chi\right) \circ \phi^{-1} \\
& \tilde{b}^{k}:=\left(g^{i r} a_{r}^{j} \partial_{i j}^{2} \chi^{k}+\left(\sqrt{\frac{|a|}{|g|}} \partial_{i}\left(\sqrt{\frac{|g|}{|a|}} g^{k l} a_{k}^{i}\right)+b^{l}\right) \partial_{l} \chi^{k}\right) \circ \phi^{-1} \\
& \tilde{c}:=c \circ \phi^{-1}
\end{aligned}
$$

and $\tilde{A}_{i}:=\tilde{a}^{i j} \partial_{i j}^{2}+\tilde{b}^{k} \partial_{k}+\tilde{c}$ we obtain

$$
\left(A_{i} f_{i}\right) \circ \phi^{-1}=\tilde{A}_{i} \tilde{f}_{i} \text { on } V_{i}
$$

and therefore

$$
\tilde{A}_{i} \tilde{f}_{i} \geq \tilde{h}_{i} \text { on } V_{i}
$$


for $i \in\{1, \ldots, r\}$. Note that since $\phi_{i}$ are smooth diffeomorphisms and the operators $A_{i}$ are strictly elliptic, we obtain that the operators $\tilde{A}_{i}$ are elliptic. Thus our strictly elliptic partial differential equation (A.3) is the collection of finitely many strictly elliptic differential equations (A.4) on bounded sets $V_{i}$ with piese-wise smooth boundary. Since every (second-countable) manifold (with boundary) admits an adequate atlas, we can even choose $V_{i}=B_{1}(0)^{+}:=\left\{x \in B_{1}(0): x_{n} \geq 0\right\} \subset \mathbb{R}_{+}^{n}$. Further, let $f^{+}$ be the positive part of $f$. Since $\chi$ is positive, we obtain $f_{i}^{+}=\chi_{i} \cdot f^{+}$. The following weak maximum principle is a direct consequence of [24, Thm. 9.1].

Theorem A.1 Assume $c \leq 0$. Let $A_{m} f \geq$ h on $\bar{M}$ for $f \in D\left(A_{m}\right) \cap \mathrm{W}_{\text {loc }}^{2, n}(M) \cap \mathrm{C}(\bar{M})$. Then

$$
\sup _{q \in \bar{M}} f(q) \leq \sup _{q \in \partial M} f^{+}(q)+C\|h\|_{\mathrm{L}^{n}(M)},
$$

where $|\tilde{a}|:=\operatorname{det}\left(\tilde{a}^{i j}\right), C>0$. In particular for $h=0$ we obtain

$$
\sup _{q \in \bar{M}}|f(q)| \leq \sup _{q \in \partial M}|f(q)|
$$

Proof Applying the weak maximum principle [24, Thm. 9.1] to (A.4) yields

$$
\sup _{x \in V_{i}} \tilde{f}_{i}(x) \leq \sup _{x \in \partial V_{i}} \tilde{f}_{i}^{+}(x)+C\left\|\tilde{h}_{i}\right\|_{\mathrm{L}^{n}\left(V_{i}\right)}
$$

Denote by $\Gamma_{i}:=\left\{x_{n}=0\right\} \cap V_{i}$. Since $f_{i}$ vanishes on $\bar{M} \backslash V_{i}$ we obtain

$$
\sup _{x \in \partial V_{i}} \tilde{f}_{i}^{+}(x)=\sup _{x \in \Gamma_{i}} \tilde{f}_{i}^{+}(x)
$$

We conclude

$$
\sup _{q \in U_{i}} f_{i}(q) \sup _{x \in V_{i}} \tilde{f}_{i}(x) \leq \sup _{x \in \Gamma_{i}} \tilde{f}_{i}^{+}(x)+C\left\|\tilde{h}_{i}\right\|_{\mathrm{L}^{n}\left(V_{i}\right)}=\sup _{q \in U_{i} \cap \bar{M}} f_{i}^{+}(q)+C\left\|h_{i}\right\|_{\mathrm{L}^{n}\left(U_{i}\right)} .
$$

Using $u=\sum_{i=1}^{r} f_{i}$ and $h=\sum_{i=1}^{r} f_{i}$ the claim follows.

Since the proofs of the a priori bounds (cf. [24, Thm. 9.11, Thm. 9.13 \& Lem. 9.16]) use localization techniques they can be easily generalized to manifolds with boundary. We obtain the following result.

Theorem A.2 Let $1<p, q<\infty$ and $T \subset \partial M$ a $\mathrm{C}^{1,1}$ boundary portion. Let $f \in$ $\mathrm{W}^{2, p}(M)$ be a strong solution of $A_{m} f=h$ with $h \in \mathrm{L}^{q}(M)$ with $\left.f\right|_{T}=0$. Then $f \in \mathrm{W}^{2, q}(M)$ and for all compact subsets $K \subset M \cup T$ we have

$$
\|f\|_{\mathrm{W}^{2, q}(K)} \leq C \cdot\left(\|f\|_{\mathrm{L}^{q}(M)}+\|h\|_{\mathrm{L}^{q}(M)}\right)
$$

Now we prove unique existence of the Dirichlet problem as in [24, Cor 9.15]. 
Theorem A.3 The inhomogeneous Dirichlet problem

$$
\left\{\begin{array}{l}
A_{m} f=h \\
\left.f\right|_{\partial M}=\varphi
\end{array}\right.
$$

for $h \in \mathrm{L}^{p}(M)$ and for $\varphi \in \mathrm{W}^{2, p}(M)$ with $p \in(1, \infty)$ admits a unique solution $f \in \mathrm{W}^{2, p}(M)$.

Proof By considering $f-\varphi$ we can assume without loss of generality $\varphi=0$. Since $f_{i}$ vanishes outside of $U_{i}$ and on $U_{i} \cap \partial M$ we conclude that $\tilde{f}_{i}=0$ on $\partial M$. From (A.4) it follows that

$$
\left\{\begin{array}{l}
\tilde{A}_{i} \tilde{f}_{i}=0 \text { on } V_{i} \\
\tilde{f}_{i}=0 \text { on } \partial V_{i} .
\end{array}\right.
$$

To apply [24, Thm 9.15] we need a $C^{1,1}$-domain, but $V_{i}=\left\{x \in B_{1}(0): x_{n} \leq 0\right\}$ is not a $\mathrm{C}^{1,1}$-domain. Using the extension operator

$$
\left(E \tilde{f}_{i}\right)(x):= \begin{cases}\tilde{f}_{i}(x) & \text { for } x_{n} \geq 0 \\ -\tilde{f}_{i}\left(x_{1}, \ldots, x_{n-1},-x_{n}\right) & \text { for } x_{n}<0\end{cases}
$$

for $x \in B_{1}(0)$. We extend $\tilde{A}_{i}$ to $\hat{A}_{i}:=\hat{a}^{i j} \partial_{i j}^{2}+\hat{b}^{k} \partial_{k}+\hat{c}$ with

$$
\begin{aligned}
\hat{a}^{i j} & := \begin{cases}\tilde{a}^{i j}(x) & \text { for } x_{n} \geq 0, \\
\left.(-1)^{\left\lfloor\frac{i}{n}\right\rfloor}(-1)^{\left\lfloor\frac{j}{n}\right.}\right\rfloor \tilde{a}^{i j}\left(x_{1}, \ldots, x_{n-1},-x_{n}\right) & \text { for } x_{n} \leq 0,\end{cases} \\
\tilde{b}^{k} & := \begin{cases}\hat{b}^{k}(x) & \text { for } x_{n} \geq 0, \\
(-1)^{\left\lfloor\frac{k}{n}\right\rfloor} \tilde{b}^{k}\left(x_{1}, \ldots, x_{n-1},-x_{n}\right) & \text { for } x_{n} \leq 0,\end{cases} \\
\tilde{c} & := \begin{cases}\hat{c}(x) & \text { for } x_{n} \geq 0, \\
\tilde{c}\left(x_{1}, \ldots, x_{n-1},-x_{n}\right) & \text { for } x_{n} \leq 0 .\end{cases}
\end{aligned}
$$

Then $\hat{a}^{i j}$ is strictly elliptic on $B_{1}(0)$ and symmetric. Note that all coefficients are $\mathrm{L}^{\infty}$ functions on $B_{1}(0)$ and $\operatorname{osc}\left(\hat{a}^{i j}\right)(x):=\inf _{\rho>0} \sup _{y, z \in B_{\rho}(x)}\left|a^{i j}(y)-a^{i j}(z)\right|<\varepsilon$ for all $x \in B_{1}(0)$. Moreover $E \tilde{f}_{i}$ satisfies the elliptic equation

$$
\begin{cases}\hat{A}_{i} E \tilde{f}_{i} & =E \tilde{h}_{i} \text { on } B_{1}(0) \\ E \tilde{f}_{i} & =0 \text { on } \partial B_{1}(0)\end{cases}
$$

with $E \tilde{h}_{i} \in \mathrm{L}^{p}\left(B_{1}(0)\right)$. Note that $B_{1}(0)$ is smooth and we can now apply [24, Thm. 9.15]. This implies the unique existence of a solution $E \tilde{f}_{i} \in \mathrm{W}^{2, p}\left(B_{1}(0)\right)$ of (A.6). Since $\left.\tilde{f}_{i}\right|_{\partial V_{i}}=0$ we conclude $\$ \tilde{f}_{i} \in \mathrm{W}^{2, p}\left(V_{i}\right)$ is the unique solution of (A.5). Hence

$$
\left\{\begin{array}{l}
A_{i} f_{i}=h_{i} \text { on } U_{i} \\
f_{i}=0 \text { on } \partial U_{i}
\end{array}\right.
$$


admits a unique solution $f_{i}=\tilde{f}_{i} \circ \phi \in \mathrm{W}^{2, q}\left(U_{i}\right)$ and the claim follows from $f=$ $\sum_{i=1}^{r} f_{i}$.

Using the same approximation technique as [24, Section 9.6] we conclude the following statement.

Corollary A.4 The inhomogeneous Dirichlet problem

$$
\left\{\begin{array}{l}
A_{m} f=h \\
\left.f\right|_{\partial M}=\varphi
\end{array}\right.
$$

for $h \in \mathrm{L}^{p}(M)$ and $\varphi \in \mathrm{C}(\partial M)$ admits a unique solution $f \in \mathrm{C}(\bar{M}) \cap \mathrm{W}_{\text {loc }}^{2, p}(M)$.

Proof For the uniqueness consider two solution $f_{1}, f_{2} \in \mathrm{C}(\bar{M}) \cap \mathrm{W}_{\text {loc }}^{2, p}(M)$. Then $f_{1}-f_{2} \in \mathrm{C}(\bar{M}) \cap \mathrm{W}_{\text {loc }}^{2, p}(M)$ satisfies

$$
\left\{\begin{array}{l}
A_{m}\left(f_{1}-f_{2}\right)=0 \\
\left.\left(f_{1}-f_{2}\right)\right|_{\partial M}=0
\end{array}\right.
$$

Now the maximum principle (Theorem A.1) implies $f_{1}=f_{2}$.

For the existence choose a sequence $\left(\varphi_{l}\right)_{l \in \mathbb{N}} \subset \mathrm{W}^{2, p}(M)$ with $\varphi_{l} \rightarrow \varphi$ in $\mathrm{C}(\partial M)$. By Theorem A.3 there exists unique solution $f_{l} \in \mathrm{W}^{2, p}(M)$ of

$$
\left\{\begin{array}{l}
A_{m} f_{l}=h \\
\left.f_{l}\right|_{\partial M}=\varphi_{l} .
\end{array}\right.
$$

The differences $\left(f_{l}-f_{k}\right)$ satisfies

$$
\left\{\begin{array}{l}
A_{m}\left(f_{l}-f_{k}\right)=0 \\
\left.\left(f_{l}-f_{k}\right)\right|_{\partial M}=\varphi_{l}-\varphi_{k} .
\end{array}\right.
$$

Now the a priori bounds (Theorem A.2) and the maximum principle (Theorem A.1) yields

$\left\|f_{l}-f_{k}\right\|_{\mathrm{W}^{2, p}(K)} \leq C \cdot\left\|f_{l}-f_{k}\right\|_{\mathrm{L}^{p}(M)} \leq C \cdot\left\|f_{l}-f_{k}\right\|_{\mathrm{C}(\bar{M})}=C \cdot\left\|\varphi_{l}-\varphi_{k}\right\|_{\mathrm{C}(\partial M)}$

for all compact subsets $K \subset M$. Hence $\left(f_{l}\right)_{l \in \mathbb{N}}$ converges in $\mathrm{C}(\bar{\Omega}) \cap \mathrm{W}_{\text {loc }}^{2, p}(\Omega)$ to a solution $f \in \mathrm{C}(\bar{\Omega}) \cap \mathrm{W}_{\text {loc }}^{2, p}(\Omega)$ of the Dirichlet problem (A.7).

\section{References}

1. Arendt, W., Batty, C.J.K., Hieber, M., Neubrander, F.: Vector-Valued Laplace Transforms and Cauchy Problems, Birkhäuser, Basel (2011) 
2. Adams, R.A.: Sobolev Spaces. Academic Press, New York (1975)

3. Amann, H.: Linear and Quasilinear Parabolic Problems, vol. 1, Birkhäuser (1995)

4. Amann, H., Escher, J.: Strongly continuous dual semigroups. Ann. Mat. Pura Appl. 171, 41-62 (1996)

5. Arendt, W., ter Elst, A.F.M.: The Dirichlet-to-Neumann operator on rough domains. J. Differ. Equ. 251, 2100-2124 (2011)

6. Arendt, W., ter Elst, A.F.M.: The Dirichlet-to-Neumann operator on C( $\partial \Omega)$. Ann. Scuola Norm. Sup. Pisa Cl. Sci. 20, 1169-1196 (2020)

7. Arendt, W., Elst, A.F.M., Kennedy, J.B., Sauter, M.: The Dirichlet-to-Neumann operator via hidden compactness. J. Funct. Anal. 266, 1757-1786 (2014)

8. Arendt, W., Metafune, G., Pallara, D., Romanelli, S.: The Laplacian with Wentzell-Robin boundary conditions on spaces of continuous functions. Semigroup Forum 67, 247-261 (2003)

9. Binz, T., Engel, K.-J.: Operators with Wentzell boundary conditions and the Dirichlet-to-Neumann operator. Math. Nachr. 292, 733-746 (2019)

10. Binz, T.: Strictly elliptic perators with Dirichlet boundary conditions on spaces of continuous functions on manifolds. J. Evol. Equ. 20, 1005-1028 (2020)

11. Casarino, V., Engel, K.-J., Nagel, R., Nickel, G.: A semigroup approach to boundary feedback systems. Integral Equ. Oper. Theory 47, 289-306 (2003)

12. Casarino, V., Engel, K.-J., Nickel, G., Piazzera, S.: Decoupling techniques for wave equations with dynamic boundary conditions. Discrete Contin. Dyn. Syst. 12, 761-772 (2005)

13. Campiti, M., Metafune, G.: Ventcel's boundary conditions and analytic semigroups. Arch. Math. 70, 377-390 (1998)

14. Clément, P., Timmermans, C.A.: On $C_{0}$-semigroups generated by differential operators satisfying Ventcel's boundary conditions. Indag. Math. 89, 379-387 (1986)

15. ter Elst, A.F.M., Ouhabaz, E.M.: Analysis of the heat kernel of the Dirichlet-to-Neumann operator. J. Funct. Anal. 267, 4066-4109 (2014)

16. ter Elst, A.F.M., Ouhabaz, E.M.: Analyticity of the Dirichlet-to-Neumann semigroup on continuous functions. J. Evol. Equ. 19, 21-31 (2019)

17. Engel, K.-J., Fragnelli, G.: Analyticity of semigroups generated by operators with generalized Wentzell boundary conditions. Adv. Differ. Equ. 10, 1301-1320 (2005)

18. Engel, K.-J., Nagel, R.: One-Parameter Semigroups for Linear Evolution Equations. Springer, Berlin (2000)

19. Escher, J.: The Dirichlet Neumann operator on continuous function. Annali della Scuola Normale Superiore di Pisa 21, 235-266 (1994)

20. Engel, K.-J.: The Laplacian on $C(\bar{\Omega})$ with generalized Wentzell boundary conditions. Arch. Math. 81, 548-558 (2003)

21. Favini, A., Goldstein, G., Goldstein, J.A., Obrecht, E., Romanelli, S.: Elliptic operators with general Wentzell boundary conditions, analytic semigroups and the angle concavity theorem. Math. Nachr. 283, 504-521 (2010)

22. Favini, A., Goldstein, G., Goldstein, J.A., Romanelli, S.: General Wentzell boundary conditions, differential operators and analytic semigroups in $C[0,1]$. Bol. Soc. Parana. Mat. 20, 93-103 (2002)

23. Feller, W.: Diffusion processes in one dimension. Trans. Am. Math. Soc. 97, 1-31 (1954)

24. Gilbarg, D., Trudinger, N.S.: Elliptic Partial Differential Equations of Second Order, Springer, Berlin (2001) Reprint of the 1998 edition

25. Hebey, E.: Sobolev Spaces on Riemannian Manifolds. Springer, Berlin (1996)

26. Greiner, G.: Perturbing the boundary conditions of a generator. Houston J. Math. 13, 213-229 (1987)

27. Hebey, E.: Nonlinear Analysis on Manifolds: Sobolev Spaces and Inequalities. Am. Math. Soc. (2000)

28. Holderrieth, A.: Multiplicative Perturbations. Universität Tübingen, Diss (1992)

29. Lassas, M., Taylor, M.E., Uhlmann, G.: The Dirichlet-to-Neumann map for complete Riemannian manifolds with boundary. Commun. Geom. Anal. 11, 207-221 (2003)

30. Lassas, M., Uhlmann, G.: On determining a Riemannian manifold from the Dirichlet-to-Neumann map. Ann. Sci. Ecole Norm. Sup. 34, 771-787 (2001)

31. Lorenzi, L., Rhandi, A.: Semigroups of Bounded Operators and Second-Order Elliptic and Parabolic Partial Differential Equations. Taylor \& Francis Group (2021)

32. Lunardi, A.: Analytic Semigroups and Optimal Regularity in Parabolic Problems. Birkhäuser, Basel (1995)

33. Renardy, M., Rogers, R.C.: An Introduction to Partial Differential Equations. Springer, Berlin (2004)

34. Taylor, M.E.: Pseudodifferential Operators. Princeton University Press, Princeton (1981) 
35. Taylor, M.E.: Partial Differential Equations II. Springer, Berlin (1996)

36. Uhlmann, G., Sylvester, J.: The Dirichlet to Neumann map and applications. In: Colton, D., Ewing, R., Rundell, W. (eds.), Inverse Problems in Partial Differential Equations. Proceedings of the Conference Held at Humboldt State University, Arcata, CA, July 29-August 4, 1989, SIAM, Philadelphia, PA, pp. 101-139 (1990)

37. Vogt, H., Voigt, J.: Wentzell boundary conditions in the context of Dirichlet forms. Adv. Differ. Equ. 8, 821-842 (2003)

38. Warma, M.: Analyticity on $L_{1}$ of the heat semigroup with Wentzell boundary conditions. Arch. Math. 94, 85-89 (2010)

39. Wentzell, A.D.: On boundary conditions for multidimensional diffusion processes. Theor. Probab. Appl. 4, 164-177 (1959)

Publisher's Note Springer Nature remains neutral with regard to jurisdictional claims in published maps and institutional affiliations. 